\title{
Conservación de las aves amenazadas que habitan en los Pantanos de Villa, Lima, Perú
}

Conservation of threatened birds inhabiting over Pantanos de Villa, Lima, Peru

\author{
(D) Víctor Pulido Capurro ${ }^{1} \square$, (D) Edith Olivera Carhuaz², (D) Edith Farías Jiménez", \\ (iD) Antuanet Chirinos Mendoza ${ }^{1}$, (D) Antonio Reynaga Alponte ${ }^{3}$, (D) Ricardo Ruiz Villavicencio ${ }^{4}$
}

${ }^{1}$ Universidad Privada San Juan Bautista. Lima, Perú

${ }^{2}$ Universidad Jaime Bausate y Meza. Lima, Perú

${ }^{3}$ Universidad Tecnológica del Perú. Lima, Perú

${ }^{4}$ Universidad Cesar Vallejo. Lima, Perú

Recibido: $23 / 10 / 2020 \quad$ Revisado: $17 / 11 / 2020 \quad$ Aceptado: $23 / 11 / 2020 \quad$ Publicado: $16 / 12 / 2020$

\section{RESUMEN}

En el Refugio de Vida Silvestre Pantanos de Villa ubicado en la costa central sobre una extensión de 263,27 ha, habitan 211 especies de aves de las cuales oficialmente 19 especies están categorizadas como amenazadas. Se describe el estado actual de la conservación de las especies de aves amenazadas, rango de distribución y tendencia de sus poblaciones, así como se destaca la importancia de los hábitats de los Pantanos de Villa como lugar de refugio y conservación de especies en el Perú. Palabras clave: Pantanos de Villa, refugio de vida silvestre, especies amenazadas, peligro de extinción, perdida de hábitats, humedales.

\section{ABSTRACT}

In the Wildlife Refuge Villa Swamps located in the central coast on an area of 263.27 ha, there were 211 species of birds of which officially 19 species are categorized as threatened. The current state of the conservation of threatened bird species, the range of distribution and the trend of relationships are described, as well as the importance of the habits of the Villa Swamps as a place of refuge and conservation of the species in Peru. Keywords: Villa swamps, wildlife refuge, endangered species, danger of extinction, loss of habitats, wetlands. 


\section{INTRODUCCIÓN}

La extinción de especies de plantas y animales, como elementos esenciales de la biodiversidad asociadas a la "sexta extinción masiva", constituye un obstáculo difícil de eludir (Ceballos et al., 2011). Se estima que, en la Tierra, en los últimos 250 millones de años deben haberse extinguido entre el $77 \%$ y el $96 \%$ del total de especies (Raup, 1979). Myers (1979) señala que, al menos el $90 \%$ de las especies han desaparecido. La mayoría de los autores coinciden que entre los 15,000 a 50,000 años, el hombre ha tenido un rol determinante en la extinción de las especies. De acuerdo a las listas rojas de UICN (2019) de las 63,837 especies evaluadas 19.817 están amenazadas por la extinción, incluyendo el $41 \%$ de anfibios, $33 \%$ de los corales de arrecifes, $25 \%$ de mamíferos, $13 \%$ de aves y $30 \%$ de coníferas. Según Ceballos et al. (2010), cada cien años se extingue una entre 10,000 especies, en tanto la tasa de extinción, tomando como referencia el registro histórico de las listas de la IUCN es unas 6.500 veces más acelerada. A nivel de la región neotropical, la tasa de extinción de vertebrados terrestres es de 280 veces más acelerada (Ceballos y Ortega-Baes, 2011).

Las aves conforman el grupo de vertebrados terrestres con mayor riqueza de especies en el mundo, con alrededor de 10507 especies (Gill \& Donsker, 2019). No obstante, de acuerdo a BirdLife International (2018) desde el año 1500, un total de 161 especies de aves se han extinguido; lo que incluye cinco especies que se han extinguido en la naturaleza, pero todavía se mantienen poblaciones en cautiverio; 22 especies están clasificadas en peligro crítico, aunque podrían estar extintas, y 183 especies como posiblemente extintas en los últimos 500 años. En el Perú de un total de 1861 especies (Plenge, 2018), 190 especies de aves sil- vestres se encuentran categorizadas como especies amenazadas y en peligro de extinción.

En este escenario, el Refugio de Vida Silvestre de los Pantanos de Villa, que forma parte del Sistema Nacional de Áreas Protegidas es uno de los humedales más importantes en la costa central del Perú, donde se protege parte de la biodiversidad de la ecorregión del desierto Costero (SERNANP, 2016). De las 211 especies de aves reportadas en Pantanos de Villa (Pulido, 2018), 19 especies de aves están amenazadas (Perú, 2014). El objetivo del presente estudio es describir el estado actual de las especies de aves amenazadas que residen en los hábitats de los Pantanos de Villa y destacar su importancia como lugar de refugio y conservación de especies en el Perú.

\section{MATERIAL Y MÉTODOS}

\section{Área de estudio}

El Refugio de Vida Silvestre de los Pantanos de Villa se encuentran situados al sur de la ciudad de Lima, en el distrito de Chorrillos, Lima, en la ecorregión del Desierto Costero y Geográficamente se encuentra entre las coordenadas $12^{\circ} 10^{\prime}-12^{\circ} 13^{\prime} \mathrm{S}$; $77^{\circ} 01^{\prime}-77^{\circ} 02^{\prime}$ W abarcan una extensión de 263,27 ha. La altitud varía desde el nivel del mar hasta los 5 $\mathrm{m}$, siendo la profundidad máxima de $1,5 \mathrm{~m}$ en los espejos de agua. La precipitación mensual oscila entre $0,0 \mathrm{~mm}$ y $5,5 \mathrm{~mm}$; y la temperatura media mensual presenta valores máximos en marzo con $25,8{ }^{\circ} \mathrm{C}$ y mínimos $15,6^{\circ} \mathrm{C}$ en setiembre. La humedad relativa media es de $86 \%$; la evaporación oscila entre $30 \mathrm{~mm} / \mathrm{mes}$, de junio a setiembre y $225 \mathrm{~mm} / \mathrm{mes}$, de enero a marzo. Los valores máximos de horas de sol son de 9 horas y los mínimos de 0,5 horas (Young 1998; Pulido y Bermúdez, 2018). 


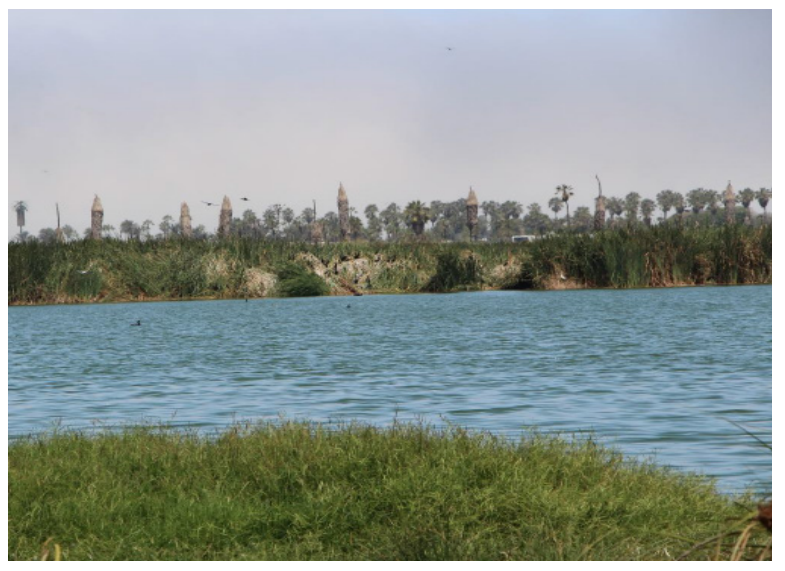

Figura 1. Vista panorámica de las lagunas en pantanos de Villa

\section{Identificación de especies}

El registro de las especies de aves se realizó desde el año 1973 hasta el 2019, en los hábitats presentes en los pantanos de Villa, determinados por Pulido \& Bermúdez (2018) y son los siguientes: laguna con espejo de agua, totoral, zona arbustiva, vega de ciperáceas, gramadales, canales y depresiones, litoral marino, parques y jardines. Las observaciones de aves fueron efectuadas con binoculares (7 x 35); y se utilizaron las Guías de campo de Koepcke (1964), Schulenberg et al. (2010) "Aves de Perú", y para la clasificación taxonómica se siguió a Plenge (2020), en "List of the birds of Peru".

\section{RESULTADOS Y DISCUSIÓN}

\section{La categorización de especies}

La Ley $N^{\circ} 27308$ regula el manejo y aprovechamiento a nivel nacional de todas las especies y establece que cada tres años, se actualice la clasificación de especies de flora y fauna silvestre, con respecto a su estado de conservación, teniendo en cuenta procedimientos internacionalmente reconocidos, como es el caso de los criterios y categorías de la Unión Internacional para la Conservación de la Naturaleza (UICN), para definir medidas de protección y aprovechamiento sostenible. El proceso de categorización de la lista de especies de fauna silvestre amenazada del Perú, utiliza información sobre población, amenazas a los taxones de poblaciones silvestres, dentro de su ámbito de distribución natural; para sobre la base de los criterios y el riesgo de extinción de los taxones clasificarlos según su grado de amenaza (Perú, 2014). En los últimos 43 años, en el Perú se han realizado cinco categorizaciones (1977, 1990, 1999, 2004, 2014), han comprendido de 104 a 190, 222, 301 y 535 especies de fauna silvestre (Tabla 1) consideradas amenazadas y en peligro de extinción (Pulido, 2009, Perú 2014).

Tabla 1. Número de especies en las categorizaciones de especies de aves amenazadas efectuadas en 1977, 1990 y 1999, 2004 y 2014, para los Pantanos de Villa

\begin{tabular}{|l|l|l|l|l|l|}
\hline $\begin{array}{l}\text { Año de la } \\
\text { categorización }\end{array}$ & $\begin{array}{l}\text { Total especies } \\
\text { amenazadas }\end{array}$ & $\begin{array}{l}\text { Total especies } \\
\text { amenazadas } \\
\text { de aves }\end{array}$ & $\begin{array}{l}\text { Especies } \\
\text { amenazadas } \\
\text { de aves en } \\
\text { Villa }\end{array}$ & $\begin{array}{l}\text { Porcentaje de } \\
\text { total de } \\
\text { especies de } \\
\text { fauna } \\
\text { amenazadas }\end{array}$ & $\begin{array}{l}\text { Porcentaje de } \\
\text { total de especies } \\
\text { de aves } \\
\text { amenazadas }\end{array}$ \\
\hline 1997 & 104 & 33 & 9 & 8.65 & 27.27 \\
\hline 1990 & 170 & 69 & 22 & 12.94 & 31.88 \\
\hline 1999 & 222 & 86 & 23 & 10.45 & 26.74 \\
\hline 2004 & 301 & 172 & 19 & 6.31 & 11.05 \\
\hline 2014 & 535 & 190 & 19 & 3.55 & 10.00 \\
\hline
\end{tabular}


En los últimos 43 años, en el Perú se han realizado cinco categorizaciones (1977, 1990, 1999, 2004, 2014), han comprendido de 104 a 190, 222, 301 y 535 especies de fauna silvestre (Tabla 1) consideradas amenazadas y en peligro de extinción (Pulido, 2009, Perú 2014). La primera categorización de especies de 1977, incluyó a 104 especies de fauna; de las cuales 33 especies fueron especies de aves y 9 especies consideradas en situación vulnerable habitan en Villa; lo que significaba el $27.27 \%$ del total de especies de aves amenazadas del Perú (Pulido, 1991).

La segunda categorización de especies aprobada en 1990, incluyó 170 especies; 69 especies fueron de aves, de las cuales 22 habitan en Villa: 2 en vías de extinción, 13 vulnerables, 5 raras y 2 indeterminadas; lo que significaba el $31.88 \%$ del total de especies de aves amenazadas del Perú (Pulido, 1991).

La tercera categorización se aprobó en 1999, con 222 especies de fauna de las que 86 fueron aves; 23 especies de aves se encontra ron en Villa: una en peligro, 15 en situación vulnerable, 5 en situación rara y 2 en situación indeterminada; lo que significaba el 26.74\% del total de especies de aves amenazadas del Perú (Pulido, 2009).

La cuarta categorización que se aprobó en 2004, incluyó a 301 especies de fauna, de este total, 172 fueron aves, 19 especies se encontraron en Villa: una en estado crítico, 8 en peligro, 4 en vulnerables y 6 en casi amenazado; lo que significaba el $11.05 \%$ del total de especies de aves amenazadas del Perú (Pulido, 2009).

La quinta categorización que se aprobó en 2014, incluye a 535 especies, 190 son de aves, de las cuales 19 se encuentran en Villa: 2 se encuentran en estado crítico, 5 en peligro, 5 en situación vulnerable y 7 en casi amenazado; lo que significaba el $10.00 \%$ del total de especies de aves amenazadas del Perú; lo que le confiere un valor especial a este refugio de vida silvestre (Perú, 2014).

En esa línea de tiempo, el número de especies de aves amenazadas en Pantanos de Villa ha variado de 9 a 22 y 23 especies, en el periodo de 1977, 1990 a 1999; y se estabiliza con 19 especies en el periodo 2004 a 2014 (Tabla 2).

Tabla 2. Comparación de las categorizaciones de especies de aves amenazadas efectuadas en 1977, 1990 y 1999, 2004 y 2014, para los Pantanos de Villa

$\begin{array}{llllll}\text { Especies } & \mathbf{1 9 7 7} & \mathbf{1 9 9 0} & \mathbf{1 9 9 9} & \mathbf{2 0 0 4} & \mathbf{2 0 1 4} \\ \text { Podiceps occipitalis } & - & - & - & \text { NT } & \text { NT } \\ \text { Laterallus jamaicensis } & - & - & - & \text { NT } & \text { CR } \\ \text { Daption capensis } & - & \text { R } & \text { R } & - & - \\ \text { Pelecanoides garnotii } & \text { V } & \text { E } & \text { E } & \text { CR } & \text { VU } \\ \text { Oceanodroma markhami } & - & - & - & \text { EN } & \text { VU } \\ \text { Pelecanus thagus } & - & - & \text { V } & \text { EN } & \text { EN } \\ \text { Sula nebouxii } & \text { V } & \text { V } & \text { V } & - & - \\ \text { Sula variegata } & \text { V } & \text { V } & \text { V } & \text { EN } & \text { EN } \\ \text { Phalacrocorax bouganvillii } & \text { V } & \text { V } & \text { V } & \text { EN } & - \\ \text { Phalacrocorax gaimardi } & \text { V } & \text { V } & \text { V } & \text { EN } & \text { EN } \\ \text { Phalacrocórax olivaceus } & \text { V } & \text { V } & \text { V } & - & - \\ \text { Theristicus melanopis } & - & \text { V } & \text { V } & - & \text { VU } \\ \text { Platalea ajaja } & \text { V } & \text { V } & \text { V } & \text { EN } & \text { NT }\end{array}$


Phoenicopterus chilensis

Dendrocygna autumnalis

Sarkidiornis melanotos

Falco peregrinus

Fulica gigantea

Fulica rufifrons

Haematopus ater

Haematopus palliatus

Recurvitostra andina

Larus dominicanus

Larus serranus

Larosterna inca

Spheniscus humboldti

Rynchops niger

Sterna hirundinacea

Sternula lorata

Mycteria americana

Jabiru mycteria

Total

CR: en peligro crítico

E, EN: en peligro

VU: vulnerable

NT: cercanamente amenazada

R: rara

I: indeterminada

\section{a. Especies amenazadas en todas las catego- rizaciones}

- Potoyunco Pelecanoides garnotii, especie endémica del Mar Frío de la Corriente de Humboldt; se distribuye desde las islas lobos de Tierra en Piura hasta Ancud en las Islas de Chiloe en Chile. Su situación desde el siglo pasado ha sido crítica; desde 1977 fue clasificada como vulnerable (Pulido, 1991), en 1990 y 1999 como en peligro, en el 2004 en estado crítico y en el 2014 como vulnerable.

- Piquero peruano Sula variegata, se distribuye en Colombia, Ecuador, Perú y Chile. Ha sido registrada en toda la costa peruana, con mayor frecuencia en el Mar Frío de la Corriente de Humboldt, entre Tacna y Piura. Durante 1977, 1990 y 1999 ha

$\begin{array}{lllll}- & \text { E } & \text { V } & \text { NT } & \text { NT } \\ - & \text { I } & \text { I } & - & - \\ - & \text { I } & \text { I } & - & - \\ - & \text { V } & \text { V } & \text { NT } & \text { NT } \\ \text { V } & \text { V } & \text { V } & \text { NT } & \text { NT } \\ - & \text { R } & \text { R } & \text { NT } & \text { VU } \\ - & R & R & - & - \\ - & \text { R } & \text { R } & - & - \\ \text { V } & \text { R } & \text { R } & - & - \\ - & \text { V } & \text { V } & - & - \\ - & \text { V } & \text { V } & - & - \\ - & \text { V } & \text { V } & \text { VU } & \text { VU } \\ - & - & - & \text { EN } & \text { EN } \\ - & \text { V } & \text { V } & - & - \\ - & - & - & \text { VU } & \text { CR } \\ - & - & - & \text { VU } & \text { EN } \\ - & - & - & \text { EN } & \text { NT } \\ - & - & - & \text { VU } & \text { NT } \\ \mathbf{9} & \mathbf{2 2} & \mathbf{2 3} & \mathbf{1 9} & \mathbf{1 9}\end{array}$

sido clasificada como vulnerable, en el 2004 y en el 2014 como en peligro.

- Chuita Phalacrocorax gaimardi, se distribuye en Argentina, Chile y Perú. Se le ha registrado en la costa peruana, entre Isla Foca (Piura) y Tacna; y sus poblaciones en el litoral y en las islas guaneras son afectadas por el fenómeno de El Niño. Desde 1977, 1990 y 1999 ha sido clasificada como vulnerable, en el 2004 y en el 2014 como en peligro.

- Espátula rosada Platalea ajaja, se distribuye en Argentina, Ecuador, Bolivia, Chile y Perú. En 1977, 1990 y 1999 ha sido clasificada como vulnerable, en el 2004 como en peligro y en el 2014 como cercanamente amenazada. 
- Gallareta gigante Fulica gigantea, se distribuye en Argentina, Bolivia, Chile, y Perú. En el Perú en ambientes acuáticos de la región andina y costera. Durante 1977, 1990 y 1999 ha sido clasificada como vulnerable, en el 2004 y en el 2014 como cercanamente amenazada.

\section{b. Especies amenazadas en las cuatro últi- mas categorizaciones}

- Flamenco Phoenicopterus chilensis, de amplia distribución en Argentina, Bolivia, Chile, Paraguay, Perú y Uruguay, en el sur de Brasil existen poblaciones migrantes no reproductivas; se han observado poblaciones en Ecuador y en las islas Malvinas. Desde 1990 ha sido clasificada como en peligro y en 1999 ha sido clasificada como vulnerable, en el 2004 y en el 2014 como cercanamente amenazada.

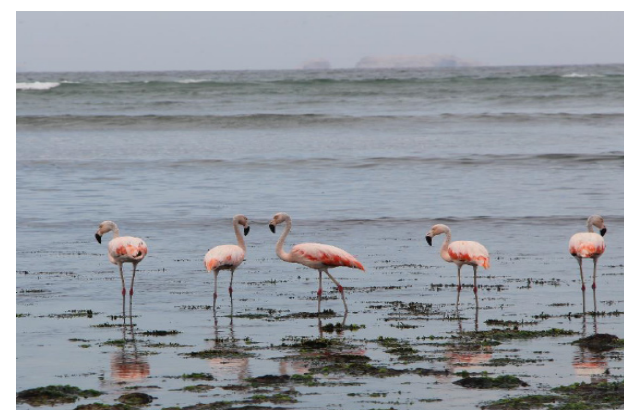

Figura 2. Flamenco Phoenicopterus chilensis en busca de alimentos

- Halcón peregrino Falco peregrinus es una especie de amplia distribución en el Perú, se considera que es migratoria Neartica aunque en los últimos años se han identificado algunas poblaciones residentes; su rango de distribución altitudinal es entre los cero hasta los $4300 \mathrm{msnm}$ (Pulido et al., 2013). Desde 1990 a 1999 ha sido clasificada como vulnerable, en el 2004 y en el 2014 como cercanamente amenazada.
- Gallareta de frente roja Fulica rufifrons, de distribución restringida a Perú y Chile. Solo se registran poblaciones en el Santuario Nacional Lagunas de Mejía, en el departamento de Arequipa y en el Refugio de Vida Silvestre de los Pantanos de Villa desde 1994 (Guillén \& Barrio, 1994). En 1990 y 1999 ha sido clasificada como rara, en el 2004 como cercanamente amenazada y en el 2014 como vulnerable

- Zarcillo Larosterna inca, habita en las costas de Chile, Colombia, Ecuador, Perú y Panamá. En el Perú, ha sido registrada a lo largo de todo el litoral, aunque las mayores concentraciones de sus poblaciones están en el Mar Frio de la Corriente de Humboldt. Desde 1990, 1999, 2004 y en el 2014 ha sido clasificada como vulnerable.

\section{c. Especies amenazadas en las tres últimas categorizaciones}

- Pelicano peruano Pelecanus thagus, habita en las costas de Chile y Perú. En el Perú, se distribuye en el mar frío, frente a la costa, influenciado por la Corriente de Humboldt; aunque también ha sido registrado en aguas tropicales de Piura y Tumbes (Schulenberg et al., 2010), aunque también ha sido visto ocasionalmente en Ecuador. Desde 1999 ha sido clasificada como vulnerable, 2004 y en el 2014 como en peligro.

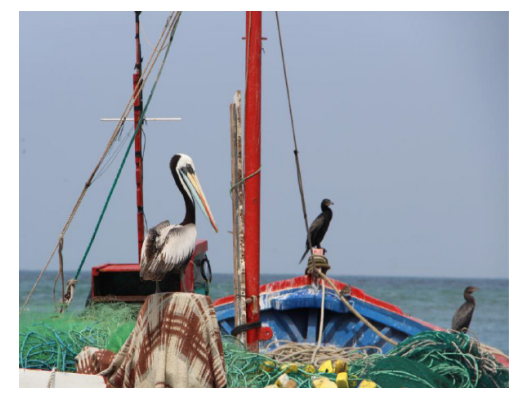

Figura 3. Pelicano Pelecanus thagus posado sobre una embarcación 


\section{d. Especies amenazadas en las dos últimas categorizaciones}

- Zambullidor plateado Podiceps occipitalis. Ampliamente distribuido en los Andes, muy raro en la Amazonia y con escasas poblaciones en la Costa; en Villa está presente en grupos de 8 a 10 ejemplares (Pulido, 2018); ha sido clasificada en el 2004 y en el 2014 como cercanamente amenazada.

- Gallineta negra Laterallus jamaicensis. Es una especie rara en el Perú, de hábitos solitarios, frecuenta los totorales y gramadales; aunque está presente durante todo el año en Villa sus poblaciones no son muy numerosas. Ha sido clasificada en el 2004 como cercanamente amenazada y en el 2014 en peligro crítico.

- Golondrina de mar de Markham Oceanodroma markhami, se estima que la población global de esta especie está alrededor de 50.000 individuos, y su tendencia se reconoce que está en declive con menos de 10000 individuos maduros (Ayala, 2018, BirdLife International, 2018). Se reproduce en las costas de Chile (Medrano et al., 2019) y en el Perú, ha sido registrada en la Reserva Nacional de Paracas y en la Reserva Nacional Sistema de Islas, Islotes y Puntas Guaneras (Ayala, 2018) y en Pantanos de Villa. Ha sido clasificada en el 2004 como en peligro y en el 2014 como vulnerable.

- Pingüino de Humboldt Spheniscus humboldti, se distribuye en las costas e islas de Perú y Chile; desde isla Foca (Perú, $5^{\circ} 12^{\prime}$ ) hasta Corral (Chile, 39 52'); y su rango reproductivo en el Perú comprende desde Isla Foca hasta Punta Coles ( $\left.17^{\circ} 42^{\prime}\right)$; se estima que en el año 2014 la población en nuestro país llegaba a los 9000 individuos para el 2014 (MacGill y Ayala, 2018); y las mayores concentraciones se encuentran en la Reserva Nacional de Paracas, en la Reserva Nacional Sistema de Islas, Islotes y Puntas Guaneras y en la Zona Reservada Illescas (BirdLife International, 2018). Visita frecuentemente las costas frente a los Pantanos de Villa. Ha sido clasificada en el 2004 y en el 2014 en peligro.

- Gaviotín sudamericano Sterna hirundinacea, de amplia distribución en Argentina, Brasil, Ecuador, Chile, Perú y Uruguay. En nuestro país solo se conoce una población reproductiva, está en la isla $\mathrm{La}$ Vieja (Ica) y se han registrado poblaciones en las aguas costeras de Huarmey, en Áncash, Villa en Lima, en San Andrés en Pisco, Ite, en Tacna (Zavalaga, 2018). Ha sido clasificada en el 2004 como vulnerable y en el 2014 en peligro crítico.

- Gaviotín peruano Sternula lorata, su distribución comprende las costas de Ecuador, Perú y Chile. En el Perú, ha sido registrada desde los manglares de San Pedro de Vice, en Piura, hasta los humedales de Ite y en Tacna (Zavalaga et al., 2009). Se han efectuado algunos registros cerca de playa la Meza (Tacna) y en los Manglares de Tumbes (BirdLife International, 2018). Ha sido clasificada en el 2004 como vulnerable y en el 2014 en peligro.

- Cigüeña Gabán Mycteria americana. Ampliamente distribuido en América Central y del del Sur (BirdLife International, 2019). En el Perú en pantanos y ríos en la Amazonia; ocasionalmente registrada en Villa. Ha sido clasificada en el 2004 en peligro crítico y en el 
2014 como cercanamente amenazada.

- Jabirú Jabiru mycteria. De amplia distribución en Argentina, Belice, Bolivia, Brasil, Colombia, Costa Rica, Ecuador, El Salvador, Guyana Francesa, Guatemala, Guyana, Honduras, México, Nicaragua, Paraguay, Perú, Surinam, Venezuela (BirdLife International, 2018). En el Perú se distribuye ampliamente en la Amazonia y en Villa ha sido reportado como visitante ocasional (Pulido, 2018). Ha sido clasificada en el 2004 como vulnerable y en el 2014 como cercanamente amenazada.

\section{e. Especies amenazadas en la última cate- gorización}

- Bandurria de cara negra Theristicus melanopis, se distribuye en Argentina, Chile y Perú. En el Perú ha sido registrada en la zona costera desde Lambayeque hasta Tacna, en diferentes hábitats como humedales, en las lagunas Ñapique y La Niña, en Piura, en la desembocadura de los ríos y monte ribereño, campos de cultivo; y adicionalmente en lomas costeras (BirdLife International, 2018; Fjeldså y Krabbe, 1990; Koepcke, 1964; Hughes, 1991; Schulenberg et al., 2010; Vizcarra y Høgsås, 2010; Suárez y More, 2015). Ha sido clasificada en el 2014 como vulnerable.

\section{Conservación del área}

En su condición de área protegida, el Refugio de Vida Silvestre Pantanos de Villa tiene una función importante en la conservación de las especies de aves. En sus hábitats se han registrado, durante 115 años, 211 especies, comprendidas en 145 géneros 50 familias y 21 órdenes (Pulido \& Bermúdez, 2018b); de las cuales 19 están amenazadas (SERFOR, 2018). Eso quie- re decir que actualmente en Villa se protege el $10 \%$ del total de especies de aves amenazadas del Perú, lo que le confiere un valor especial.

Los esfuerzos para proteger los Pantanos de Villa se remontan a 1977, cuando se declaró a los pantanos y alrededores como Parque Zonal Metropolitano $\mathrm{N}^{\circ} 25$ para fines culturales y de recreación, pasando a conformar el Sistema de Áreas Recreacionales para Lima Metropolitana. En 1989 fue declarado como Zona Reservada de los Pantanos de Villa, sobre una superficie de 396 ha (Pulido \& Bermúdez, 2018a). Desde 1997, es reconocido como Ramsar, basado en el Criterio de humedal representativo para la conservación de las aves acuáticas; y la clasificación de Áreas Protegidas de la Unión Mundial para la Conservación de la Naturaleza, la considera en la Categoría IV, que es un área protegida manejada para la conservación con intervención a nivel de gestión, así como un área importante de conservación (IBAs) para las aves (Amaro \& Goyoneche 2017). $Y$ en el 2006, se declararon 263,27 hectáreas de los Pantanos de Villa como Refugio de Vida Silvestre (SERNANP, 2016).

Si bien durante muchos años se han adoptado importantes medidas de protección, el proceso de destrucción del hábitat ha sido continuo. Desde 1943, se han perdido 1266 ha de pantanos, por lo tanto, la tasa anual de destrucción de los pantanos ha sido de 16,8 ha/año Además la tasa de desaparición de cultivos ha sido entre 1975 y 1991 de 89,5 ha/año; y entre 1991 y el 2002 de 51 ha/año (Pulido \& Bermúdez, 2018a). Como consecuencia de la reducción del área total de los pantanos, las especies han visto reducido su hábitat y por lo tanto sus poblaciones se han vuelto escasas con el correr del tiempo. Pantanos de Villa es un humedal costero de pequeña extensión y los procesos de urbani- 
zación, debido a su ubicación en la ciudad de Lima, trae como consecuencia la contaminación y destrucción del hábitat y los recursos biológicos; viene siendo usado como depósito de desechos urbanos y toda la zona de amortiguamiento tiene problemas de contaminación por el arrojo de sólidos y líquidos contaminantes.

Las presiones antropogénicas revisten especial importancia debido a que el pantano se encuentra rodeado de asentamientos humanos que lo contaminan por efecto de los efluentes, desechos sólidos, desmontes y el ruido. La fragilidad del ecosistema ha ocasionado la instauración de un proceso gradual de desertificación y depredación de especies de flora y fauna. Además, hay que añadirle, la presión de los visitantes, como turistas, estudiantes y grupos familiares, que en los últimos años se ha incrementado a notablemente.

En tal sentido quienes están sintiendo más fuertemente la pérdida de hábitats en los pantanos son las especies amenazadas y en peligro. Por ello la conservación de las especies amenazadas en los Pantanos de Villa es significativa. Actualmente en Villa se protege el $10 \%$ del total de especies de aves amenazadas del Perú, lo que le confiere un valor especial, como área de conservación a nivel nacional y resalta su representatividad biológica dentro del Sistema Nacional de Áreas Protegidas por el Estado.

Finalmente, nuestra política debe ser la conservación de hábitats y especies. Pero también considerar a la pérdida de hábitats y especies como un asunto de considerable importancia. Por ello es imperativo desarrollar las investigaciones necesarias para encontrar las soluciones a estos desafíos. De otra manera estaríamos poniendo en serio riesgo la conservación de las especies y sus hábitats.

\section{CONCLUSIONES}

En los últimos 43 años, el número de especies de aves amenazadas en Pantanos de Villa ha variado de 9 a 22 y 23 especies, en el periodo de 1977, 1990 a 1999; a 19 especies en el periodo 2004 a 2014, lo que demuestra que las amenazas sobre el ecosistema y las especies persisten.

La quinta categorización de especies de fauna silvestre aprobada en 2014, comprende 535 especies, de las cuales 190 son de aves, y de ese total 19 se encuentran en Villa: 2 se encuentran en estado crítico, 5 en peligro, 5 en situación vulnerable y 7 en casi amenazado; lo que significaba el $10.00 \%$ del total de especies de aves amenazadas del Perú; lo que le confiere un valor especial a Pantanos de Villa como refugio de vida silvestre.

La pérdida de hábitats en los Pantanos de Villa tiene un impacto sobre las especies amenazadas y en peligro; por lo tanto los lineamientos de política para la conservación de hábitats y especies, constituyen un asunto de considerable importancia; ya que en este humedal se protege el $10 \%$ del total de especies de aves amenazadas del Perú, lo que le da un valor especial, como área de conservación y resalta su representatividad biológica dentro del Sistema Nacional de Áreas Protegidas por el Estado.

\section{REFERENCIAS BIBLIOGRÁFICAS}

Ayala, L. (2018). Oceanodroma markhami. Pp. 229.En: SERFOR. Libro Rojo de la Fauna Silvestre Amenazada del Perú. Primera edición. Lima. 548 páginas.

Amaro L. \& G. Goyoneche. (2017). Anidación de aves en el Refugio de Vida Silvestre los Pantanos de Villa 2007-2009, Lima-Perú. The Biologist (Lima), 2017, 15(1), jan-jun: 155-171. 
BirdLife International. (2016). Jabiru mycteria. The IUCN Red List of Threatened Species 2016: e.T22697710A93632239. http:// dx.doi.org/10.2305/IUCN.UK.2016-3.RLTS. T22697710A93632239.en. Downloaded on 05 July 2019.

BirdLife International. (2018). El Estado de conservación de las aves del mundo: tomando el pulso de nuestro planeta. Cambridge, Reino Unido: BirdLife International.

BirdLife International. (2019). Hoja de datos de la especie: Mycteria americana. Descargado de http://www.birdlife.org el 05/07/2019.

Ceballos, G., García, A. \& P.H. Ehrlich. (2010). The sixth extinction crisis: loss of animal populations and species. Journal of Cosmology, 8: 1821-1831.

Ceballos, G. \& P. Ortega-Baes. (2011). La sexta extinción: la pérdida de especies y poblaciones en el Neotrópico. J. Simonetti, R. Dirzo (Eds.), Conservación biológica: perspectivas de Latinoamérica, Editorial Universitaria, Chile. 95-108

Fjeldså J. \& N. Krabbe. (1990). Birds of the high Andes. Zoological Museum, University of Copenhagen and Apollo Books, Copenhagen.

Gill, F \& D Donsker (Eds). (2019). IOC World Bird List (v9.2). doi: 10.14344/IOC.ML.9.2.

Guillén C. \& J. Barrio. (1994). Los Pantanos de Villa y sus aves. Boletín de Lima, Lima. XVI (91-96): 53-58.
Hughes, R. (1991). Las aves de la Provincia de Islay. Bo $\neg$ letín de Lima 75: 47-54. IUCN (2019). The IUCN Red List of Threatened Species. Versión 2019-1. <https://www. iucnredlist.org>

Koepcke, M. (1964). Las Aves del Departamento de Lima. Edit. M. Koepcke, Lima 118 pp.

MacGill, P. \& L. Ayala. (2018). Spheniscus humboldti Pp. 270.En: SERFOR. Libro Rojo de la Fauna Silvestre Amenazada del Perú. Primera edición. Lima. 548 páginas.

Medrano, F.; Silva, R.; Barros, R.; Terán, D.; Peredo, R.; Gallardo, B. Cerpa, P.; De Groote, F.; Gutiérrez, P. \& I, Tejeda. (2019). Nuevos antecedentes sobre la historia natural y conservación de la Golondrina de mar negra (Oceanodroma markhami) y la Golondrina de mar de collar (Oceanodroma hornbyi) en Chile. Revista Chilena de Ornitología 25(1): 21-30.

Myers, N. (1979). The Sinking Ark. Pergamon Press. Inc. New York. 307 pp.

Plenge, M. (2020). List of the birds of Peru / Lista de las aves del Perú. Unión de Ornitólogos del Perú: https:77sites. google.com/site/boletinunop/checklist

Perú, (2014). Decreto Supremo que aprueba la actualización de la lista de clasificación y categorización de las especies amenazadas de fauna silvestre legalmente protegidas. Decreto Supremo No 004-2014-MINAGRI. Diario Oficial El Peruano: 520497- 520504. 
Pulido, V. (1991). El Libro Rojo de la Fauna Silvestre del Perú. INIAA, WWF, US-FWS, Ed. Mayjosa. 220 pp.

Pulido, V. (2009). Aves peruanas amenazadas y en peligro de extinción: propuestas para su conservación. Exégesis. Revista de la Escuela de Posgrado de la Universidad Inca Garcilaso de la Vega. Año 2. (2): 62-67 pp.

Pulido, V. (2018). Ciento quince años de registros de aves en Pantanos de Villa. Revista Peruana de Biología, 25(3), 291-306. doi: 10.15381/rpb.v25i3.15212.

Pulido, V. \& L. Bermúdez. (2018a). Estado actual de la conservación de los hábitats de los Pantanos de Villa, Lima, Perú. Arnaldoa 25(2), 679-702.

Pulido, V. \& L. Bermúdez. (2018b). Patrones de estacionalidad de las especies de aves residentes y migratorias de los Pantanos de Villa, Lima, Perú. Arnaldoa 25 (3): 1107-1128. DOI: http://doi.org/10.22497/arnaldoa.253.25318

Pulido, V.; Salinas, L. \& C. Arana. (2013). Aves deldesiertodelacostacentraldelPerú. Josefina Barrón Editores EIRL, Callao, Perú,pp. 1-443.

Raup, D. (1979). Size of the Permo-Triasic bottleneck ang its evolutionary implications. Science. 206: 217-218.

Schulenberg, T. S.; D. F. Stotz; D. F. Lane; J. P. O’Neill \& T. A. Parker III. (2010). Aves de Perú. Serie Biodiversidad Corbidi 01. Lima. 660 p.

SERFOR. (2018). Libro Rojo de la Fauna Silvestre Amenazada del Perú. Primera edición. SERFOR (Servicio Nacional Forestal y de Fauna Silvestre), Lima, Perú, pp 1- 548.
Suárez F. E. \& A. More. (2015). Registros la Bandurria de Cara Negra (Theristicus melanopis) en las lagunas Ñapique y La Niña, Sechura - Piura. Boletín de la Unión de Ornitólogos del Perú (UNOP), 10 (2): 31-36.

Vizcarra, J.K, \& T.E. Høgsås. (2010). Descubrimiento de dos sitios de anidamiento de Theristicus melanopis melanopis en Tacna, sur de Perú. Cotinga, No. 32: 152-153.

Young, K. (1998). El Ecosistema. En: Cano, A. y K. Young (eds.). Los Pantanos de Villa, Biología y Conservación. Museo de Historia Natural - UNMSM. Serie de Divulgación No 11: 3-20.

Zavalaga, C.; Hardesty, J.; Mori, G.; Chávez-Villavicencio, C. \& A. Tello. (2009). Current status of Peruvian Terns Sternula lorata in Peru: threats, conservation and research priorities. Bird Conservation International, 19: 175-186.

Zavalaga, C. (2018). Sterna hirundinacea Pp. 273.En: SERFOR. Libro Rojo de la Fauna Silvestre Amenazada del Perú. Primera edición. Lima. 548 páginas. 